Recepción: 01 / 03/ 2018

Aceptación: 15 / 05 / 2018

Publicación: 07 / 07 / 2018

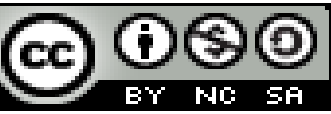

Ciencias Jurídicas

Artículo de Investigación

\title{
Perspectiva de la normativa adecuada a las pequeñas y medianas familiares industriales
}

Perspective of the appropriate regulation for industrial small and medium-

$$
\text { sized family (SMEs) }
$$

Perspectiva dos regulamentos apropriados para pequenas e médias famílias industriais

\author{
Santiago O. Ortiz-López I \\ santyomar@gmail.com \\ Kléver A. Moreno-Gavilánes II \\ kleverim@hotmail.com
}

Correspondencia: santyomar@gmail.com

I. Doctor, Carrera de Jurisprudencia, Facultad de Jurisprudencia y Ciencias Sociales, Universidad Técnica de Ambato, Ambato, Ecuador.

II Doctor, Carrera de Jurisprudencia, Facultad de Jurisprudencia y Ciencias Sociales, Universidad Técnica de Ambato, Ambato, Ecuador. 


\section{Resumen}

Las pymes en Ecuador no tienen una institucionalidad consolidada, debido a la inexistencia de una normativa específica para este sector que permitiera delimitar sus características y condiciones de operación en el ámbito económico y productivo del país, las pocas normas legales relacionadas para este sector datan de 1973, año el cual se dictó la Ley de Fomento de la Pequeña Industria. Esto ha dificultado el desarrollo e implementación eficiente de políticas públicas adecuadas que fomenten el potencial productivo del sector industrial. La metodogia utilizada en el estudio es de alcance analíticodescriptivo, ya que describe el fenómeno sucitado a raíz del problema. Finalmente, se logra visualizar que las pymes industriales son más vulnerables a los cambios que se presentan en el entorno que les rodea, poseen gran flexibilidad y capacidad de adaptación frente al cambio. Además de obtener nuevas oportunidades de crecimiento en nuevos mercados orientados a la actividad manufacturera incorporando servicios o características adicionales que le otorgan mayor valor agregado a sus productos.

Palabras claves: Normativa legal, desarrollo económico, código de comercio, sector industrial, PYMES. 


\begin{abstract}
SMEs in Ecuador do not have a consolidated institutional framework, due to the lack of specific regulations for this sector that would allow delimiting their characteristics and operating conditions in the country's economic and productive environment. The few legal regulations related to this sector date from 1973 year in which the Law of Promotion of the Small Industry was enacted. This has hindered the development and efficient implementation of adequate public policies that promote the productive potential of the industrial sector. The method used in the study is of analytical-descriptive scope, since it describes the phenomenon that has occurred as a result of the problem. Finally, it is possible to visualize that industrial SMEs are more vulnerable to the changes that occur in the environment that surrounds them, they have great flexibility and ability to adapt to change. In addition to obtaining new opportunities for growth in new markets oriented to manufacturing activity incorporating additional services or features that give greater added value to their products.
\end{abstract}

Key words: Legal regulations, economic development, commercial code, industrial sector, SMEs. 


\section{Introducción}

La necesidad de tener en conocimiento el Marco legal de las pymes se deduce a la preocupación que se genera en la cultura ecuatoriana a partir del momento de iniciar y establecer una empresa, estos argumentos se ven reflejados en la gran cantidad de empresas que son conformadas cada año. Asimismo, la dispersión de un número semejante, que se muestra influenciado en la principal causa del desconocimiento y multiplicidad de cada una de las leyes del pais.

Las pymes en Ecuador no tienen una institucionalidad consolidada, debido a la inexistencia de una normativa específica para este sector que permitiera delimitar sus características y condiciones de operación en el ámbito económico y productivo del país, las pocas normas legales relacionadas para este sector datan de 1973, año el cual se dictó la Ley de Fomento de la Pequeña Industria. Esto ha dificultado el desarrollo e implementación eficiente de políticas públicas adecuadas que fomenten el potencial productivo del sector pyme.

El gobierno propone una meta al impulso de las pequeñas y medianas empresas para transformarlas en una fortaleza motorizadora de la fracturada economía ecuatoriana. Los altos mandos de la Administración Pública han instituido apuntalar el mercado de trabajo de cada empresa a través de la promoción de las PYMES, formando parte del desarrollo que permita salir de la crisis económica que actualmente atraviesa el Ecuador.

Las pymes forman parte de los agentes importantes para la reactivación económica y productiva de un país debido a su contribución como fuentes generadoras de empleo e ingreso, además por sus características de unidades productivas que operan en menor escala que las grandes empresas, su estructura poco rígida y menor tamaño les permite adaptarse más fácilmente para atender segmentos específicos y diferenciados del mercado. Es evidente el potencial competitivo que las pymes poseen sin embargo es necesario que estas gocen de un entorno adecuado que fomente su acceso y participación en el mercado.

El presente estudio busca establecer un acercamiento hacia la realidad en la cual atraviesan las PYMES indstriales, basado en estudios anteriores, se decidió centrar el estudio en este sector, buscando así el tener una visión más cercana hacia la situación y condiciones en las que operan las pymes. 


\section{Desarrollo}

Las puqueñas y medianas empresas industriales en el país, se encuentran vulnerables a los diversos cambios que se dan en el ambiente que les envuelve, poseen gran flexibilidad y capacidad de adaptación frente al cambio; su capacidad de emprendimiento e innovación oferta varias posibilidades de alcanzar nuevos nichos de exportación para bienes no tradicionales en nuevos mercados. El sector de las pymes industriales tiene un alto potencial innovador y productivo en la generación de nuevos productos, las primordiales actividades a las que se dedican las empresas industriales están encaminadas hacia la actividad manufacturera agregando diversos servicios o características extras que le añaden un relase en el valor agregado a sus productos.

Es necesario identificar y establecer el entorno adecuado que prosiga el fortalecimiento de las pymes a través de la estabilidad económica y políticas reguladoras que fomenten el potencial productivo de las mismas. En el factor financiero, es importante tener un sistema estable y seguro que admita una intermediación financiera eficiente, donde se canalice el ahorro hacia la inversión productiva, permitiendo acceder a crédito productivo a tasas de interés y condiciones razonables.

\section{a) Marco referencial}

\section{La PYME a partir del punto jurídico.}

Cabe tener conocimiento de la conceptualizazción del término empresa, es así como Garrigues (1979) se refiere al concepto económico de empresa como la organización de los factores de la producción (capital y trabajo) con el fin de obtener una ganancia ilimitada. Los factores integrantes por la comunidad del destino económico (obtención de la ganancia máxima), constituyendo una unidad organizada conforme a las exigencias de la explotación económica. Esta unidad de concepto se manifiesta también desde el punto de vista contable, que abarca toda la actividad de la empresa dentro de determinados períodos de tiempo y separa el patrimonio mercantil del patrimonio privado del empresario (García-Moncó, 2011).

No existe un criterio estricto y único que defina a una empresa industrial como PYME, este puede verse afectado entre país y país debido a la geografía y los diferentes niveles de desarrollo de los países que exigen tomar en cuenta otros parámetros, por lo cual cada país ampara una definición de PYME acorde a su realidad. Sin embargo, existen variables que generalmente se usan para su 
medición tales como: volumen de ventas, el capital social, el número de personas ocupadas, el valor de la producción o el de los activos.

\section{Clasificación de las PYMES Industriales}

En el Ecuador no se ha establecido un criterio rígido que apruebe la conceptualización a una empresa como PYME. Sin embargo, existen criterios que generalizan a definir a una PYME con base al cumplimiento de alguno de los siguientes indicadores. Este sistema estadístico regional establece que las PYMES comprenden a todas las empresas formales legalmente constituidas y/o registradas ante las autoridades competentes, que lleven registros contables y/o aporten a la seguridad social, comprendidas dentro de los umbrales establecidos en el artículo 3 de la Decisión 702 (Cámara de Comercio de Quito, 2017).

El artículo 3 de la Decisión 702 de la CAN establece los parámetros de acuerdo a lo señalado a continuación:

a) Las empresas comprendidas dentro de los siguientes rangos de personal ocupado y de valor bruto de las ventas anuales:

\begin{tabular}{|l|c|c|c|c|}
\hline \multicolumn{1}{|c|}{ VARIABLE } & ESTRATO I & ESTRATO II & ESTRATO III & ESTRATO IV \\
\hline Personal ocupado & $1-9$ & $10-49$ & $50-99$ & $100-199$ \\
\hline Valor bruto de las & $\leq 100.000$ & $100.000-$ & $1.000 .001-$ & $2.000 .001-$ \\
ventas anuales & & 1.000 .000 & 2.000 .000 & 5.000 .000 \\
\hline
\end{tabular}

\section{Clasificación nacional}

Para lograr instituir un Estado moderno es necesario acoger políticas de protección e incentivo a la industria, por lo que se estableció leyes enfocadas a fomentar el sector industrial y al sinapismo de la pequeña industria.

La Superintendencia de Compañías, Valores y Seguros, mediante resolución, acogió la clasificación de pequeñas y medianas empresas, PYMES, de acuerdo a la normativa implantada por la Comunidad Andina en su Resolución 1260 y la legislación interna vigente, conforme al siguiente cuadro (Codificación, 1999): 


\begin{tabular}{|l|c|c|c|c|}
\hline \multicolumn{1}{|c|}{ VARIABLE } & MICROEMPRESA & $\begin{array}{c}\text { PEQUEÑA } \\
\text { EMPRESA }\end{array}$ & $\begin{array}{c}\text { MEDIANA } \\
\text { EMPRESA }\end{array}$ & $\begin{array}{c}\text { GRANDES } \\
\text { EMPRESAS }\end{array}$ \\
\hline $\begin{array}{l}\text { Personal } \\
\text { ocupado }\end{array}$ & $1-9$ & $10-49$ & $50-99$ & $100-199$ \\
\hline $\begin{array}{l}\text { Valor bruto de } \\
\text { las ventas } \\
\text { anuales }\end{array}$ & $\leq 100.000$ & $\begin{array}{c}100.000- \\
1.000 .000\end{array}$ & $\begin{array}{c}1.000 .001- \\
2.000 .000\end{array}$ & $\begin{array}{c}2.000 .001- \\
5.000 .000\end{array}$ \\
\hline $\begin{array}{l}\text { Monto de } \\
\text { activos }\end{array}$ & Hasta $\$ 100.000$ & $\begin{array}{c}\text { De } \$ 100.001 \\
\text { hasta } \$ 750.000\end{array}$ & $\begin{array}{c}\text { De } \$ 750.001 \\
\text { hasta } \$ 3.999 .999\end{array}$ & $\geq \$ 4.000 .000$ \\
\hline
\end{tabular}

\section{Periodo de industrialización sustitutiva}

A partir del año de 1950 se instauraron las primeras pymes, aquellas fueron creadas con una estructura cerrada, de origen familiar, dedicadas primariamente a actividades de tratamiento textil y confecciones, calzado y cuero e imprenta, de la misma manera minerales no metálicos. Pues a través de estas actividades precisamente habrían obtenido mayor posibilidad de establecerse los pequeños talleres personales y familiares (Elizondo M., 2009).

En 1972 el petróleo fomentó el principal rubro de exportación del país, además se intensificó el ciclo de industrialización. Sin embargo, "el proteccionismo estatal" instauro un sector industrial poco competidor que no facilitó que evolucione el mercado. Por otro lado, los empresarios conducían su preocupación por mejorar la productividad y competitividad" (Greco S., 1999).

\section{Periodo de dolarización neoliberal}

Este ajuste estructural consitia en reducir el rol del Estado por tomar en cuenta el proteccionismo estatal que no admitió que se amplíe el mercado y que los empresarios a su vez dirijan su preocupación en el mejoramiento de la productividad; por lo que se establecieron varias reformas como:

- Se le permite al mercado establecer las tasas de cambio e interés.

- La demanda y la oferta serían quienes determinen los precios.

- En el campo Fiscal se aconsejó que el gasto público disminuya y a la par se elimine los subsidios.

- Se acusó a la política Monetaria-Crediticia como causante de la inflación, por lo cual se encomendó la restricción del crédito al sector público y el alza de las tasas de interés. 
Estas situaciones obligaron a las empresas que se preocuparán únicamente por su estabilidad económica y supervivencia interna, buscando obtener los grados más altos de ingresos y productividad, generando mejores condiciones de trabajo.

\section{Marco legal de las pymes industriales}

Durante el periodo comprendido entre el año 2005 al año 2009, estuvieron vigentes:

- Ley de Fomento de la Pequeña Industria

- Ley de Comercio Exterior e Inversiones

Las normativas detalladas a continuación se encotraban relacionadas con el sector Pymes industriales. Entonces, a partir del 22 de diciembre del 2010, entró en vigencia el Código de Producción, Comercio e Inversión, el cual deja sin efecto la vigencia de las mencionadas leyes. Sin embargo, debido a que el presente estudio ha establecido como lineamiento temporal el periodo comprendido entre el año 2005 al 2009, he creído conveniente el mencionar en qué consistían las mencionadas leyes. En el Ecuador no existe una normativa específica para el sector Pyme, a continuación, se menciona las leyes relacionadas con este sector:

\section{Ley de Fomento de la Pequeña Industria:}

Fue expedida el 20 de agosto de 1973, bajo decreto $\mathrm{N}^{\circ} 921$ y publicada en Registro Oficial $\mathrm{N}^{\circ} 372$, en el gobierno del General Guillermo Rodríguez Lara.

En términos generales esta ley establece:

- Se considera Pequeña Industria a la que, con predominio de la operación de la maquinaria sobre la manual, se dedique a actividades de transformación, inclusive de forma, de materias primas o de productos semielaborados, en artículos finales o intermedios y siempre que su activo fijo, excluyendo terrenos y edificaciones, no sea mayor a 350000 dólares (monto de activos fijos reformados en Acuerdo 03 399, R.O. 151, 20-VIII2003) (Sánchez J., 2005).

- El Ministerio de Industrias y Productividad tiene como eje prioritario el apoyo y acompañamiento a los micro, pequeños y medianos empresarios para lo cual busca el desarrollar y ejecutar Programas de Fomento para estos sectores. 


\section{Beneficios}

Esta ley otorga dos tipos de beneficios: generales y específicos.

Los beneficios generales se otorgan a todas las personas naturales y jurídicas que se acogen al régimen de esta ley, es decir la pequeña industria y estos beneficios son:

- La Exoneración del pago de impuestos a actividades como:

- Constitución de Compañías

- Reforma a los estatutos de las Compañías

- Emisión, canje, fraccionamiento o conversión de los títulos, acciones o certificados de aportación

- Aumento de Capital de una Compañía

- Exoneración total de los impuestos sobre los activos totales.

- Exoneración del pago de impuestos a la importación de maquinaria, herramientas, equipos y repuestos.

- $\quad$ Exoneración de materia prima, siempre que esta no se produzca en el país.

- Percibir el 5\% como Abono Tributario sobre el valor FOB de las exportaciones. En forma alternativa las empresas acogidas a esta Ley podrán recibir el 10\% de Abono Tributario por la exportación de sus productos, porcentaje que se calculará sobre el valor agregado nacional en los productos que se exporten.

- La prohibición o limitación de la importación de artículos similares a los elaborados por la pequeña industria y artesanías nacionales, cuando estos ofrezcan condiciones satisfactorias de abastecimiento, calidad y precios.

- Las Instituciones de Crédito de Fomento están obligadas a otorgar créditos en condiciones especiales, en cuanto al monto, plazo, tasa de interés o garantía.

- El Gobierno Nacional, las instituciones públicas y privadas gocen de algún beneficio estatal, provincial, o especial, o que participen de fondos públicos, deben abastecerse de productos de la artesanía y de la pequeña industria nacional.

\section{Ley de Compañías}

Expedida el 5 de noviembre de 1999, y publicada en Registro Oficial № 312. 
Las pymes debido a su estructura pueden operar como una organización con personería jurídica o como personas naturales. Sin embargo, para aquellas que operan con personería jurídica deben acogerse a las disposiciones establecidas en la ley de compañías, la cual establece:

- La Superintendencia de Compañías es el ente regulador y de control de las compañías constituidas en el Ecuador, la Superintendencia ejerce vigilancia y control total sobre los aspectos jurídicos, societarios, económicos, financieros y contables de las empresas.

Las principales obligaciones que deben cumplir las compañías son:

- Presentar en el primer cuatrimestre de cada año:

- Copias autorizadas del balance general anual, y del estado de la cuenta de pérdidas y ganancias, aprobados por la junta general de socios o accionistas.

- Memorias e informes de los administradores y de los organismos de fiscalización establecidos por la Ley.

- La nómina de los administradores, representantes legales y socios o accionistas.

- El ejercicio económico de las compañías terminará cada 31 de diciembre.

- Toda compañía que se constituya en el Ecuador tendrá su domicilio principal dentro del territorio nacional.

\section{Ley de Comercio Exterior e Inversiones}

Ley publicada en Registro Oficial N 82, del 9 de junio de 1997.

La normativa mencionada anteriormente tiene como objetivo principal normar y promover el comercio exterior y la inversión directa en el país.

El Estado establecerá las políticas y disposiciones pertinentes para el logro de este objetivo; estas deben fundamentalmente promover los siguientes aspectos:

- Promover el crecimiento y diversificación de las exportaciones de bienes, servicios y tecnología para lograr un ritmo creciente y sostenido de desarrollo en el país.

- Aprovechar las oportunidades que brinda el comercio mundial en cuanto a tecnología, maquinaria, y otros servicios que beneficien a de la producción exportable del País. 
- Velar que el país participe en el escenario internacional conforme a prácticas leales y equitativas de libertad de comercio.

- Promover la inversión directa a través de estímulos e incentivos, así como el desarrollo de procesos de integración y el establecimiento de acuerdos comerciales bilaterales y multilaterales que amplíen la inversión y faciliten las transacciones externas del País.

- Asegurar la necesaria coherencia entre las políticas de comercio exterior y las políticas fiscal, arancelaria, monetaria, crediticia, cambiaria y de desarrollo económico - social y los correspondientes regímenes normativos.

- "Las exportaciones están exoneradas de todo impuesto, salvo las de hidrocarburos."

\section{Código Orgánico de la Producción}

El Código Orgánico de la Producción, Inversión y Comercio fue promovido durante el gobierno del Ec. Rafael Correa, el cual fue aprobado en el pleno de la Asamblea Nacional Constituyente el 22 de diciembre del 2010 y publicado en Registro Oficial N³51, el 29 de diciembre del 2010.

El objetivo primordial de este código es fomentar a los actores productivos el incentivo de la inversión por medio del establecimiento de un marco legal confiable que admita el desarrollo de la actividad productiva en el país. De esta manera, se mantendrá sujeta a su cumplimiento todas las personas naturales, jurídicas que desarrollen una actividad productiva, en cualquier parte del territorio nacional.

Por medio de la aplicación y vigencia del Código de Producción, Inversión y Comercio quedan derogadas entre otras leyes: la Ley de Fomento de la Pequeña Industria, y Ley de Comercio Exterior e Inversiones (leyes concernientes a la presente investigación). Sin embargo, el código mantiene en su mayoría los beneficios e incentivos que se encuentran establecidos en las mencionadas leyes.

Entre los nuevos incentivos que estipula el Código se puede establecer cinco tipos de incentivos:

- Incentivos Generales. - se aplica a todas las inversiones que se ejecute en cualquier parte del país, entre estos destacan:

- La reducción progresiva de tres puntos porcentuales en el impuesto a la renta.

- Deducciones adicionales para el cálculo del impuesto a la renta.

- Facilidades de pago en tributos al comercio exterior. 
- La exoneración del impuesto a la salida de divisas para las operaciones de financiamiento externo.

○ La exoneración del anticipo al impuesto a la renta por cinco años para toda inversión nueva.

- Incentivos para zonas deprimida. estas zonas se favorecen de los incentivos habituales y sectoriales antes descritos. Además, se otorga un beneficio fiscal mediante la deducción adicional del $100 \%$ del costo de contratación de nuevos trabajadores, por cinco años.

- Incentivos para las PYMES son incentivos básicamente de orden fiscal y de cofinanciamiento a las actividades de las PYMES, se determina la necesidad de establecer un régimen especial de garantías para el financiamiento privado y público de las PYMES, el cual debe ser regulado por la Superintendencia de Bancos.

- Incentivos para zonas especiales de desarrollo económico, los incentivos contemplan cero aranceles e impuestos de importaciones, arreglos laborales especiales para contratación de extranjeros y las deducciones sectoriales y territoriales.

\section{Fomento a las Micro, Pequeñas y Medianas Empresas}

El presente código establece en su capítulo III, Titulo I, Capitulo I establece los siguientes aspectos:

- Se establece el Consejo Sectorial de la Producción, como órgano regulador de las micro, pequeñas y medianas empresas -MYPYMES-. Este órgano tendrá como atribuciones y deberes principalmente:

- Aprobar las políticas, planes, programas y proyectos que incentiven el desarrollo de las PYMES.

- Establecer el presupuesto anual para la implementación de todos los programas y planes que se prioricen en su seno.

- Autorizar la creación y supervisar el desarrollo establecimientos tales como: centros de desarrollo MIPYMES, centros de investigación y desarrollo tecnológico, incubadoras de empresas, nodos de transferencia o laboratorios, requeridos para fomentar el desarrollo de este sector.

- Promover la participación de las MIPYMES en el comercio internacional mediante la coordinación con organismos especializados, públicos y privados en programas de capacitación, información, asistencia técnica y promoción comercial. 
- Propiciar la participación de universidades y centros de enseñanza en el desarrollo de programas de emprendimiento y producción a fin de fortalecer a las MIPYMES.

\section{b) Metodología}

En la presente investigación se realizó un estudio analítico-descriptivo, ya que se analizó la información levantada de las normativas adecuadas para la gestión de las pequeñas y medianas empresas (PYMES) industriales detallando el fenómeno en el cual se desenvuelve la problemática de las PYMES.

De igual manera, es de modalidad exploratoria/bibliográfica ya que porque se recolectó información de diferentes fuentes de información veraz, que realizan estudios de campo para detallar y brindar una información verídica y confiable.

\section{Conclusiones}

Las pymes industriaes en Ecuador carecen de una institucionalidad consolidada, debido a la falta de normativas específicas para este sector que permitiera delimitar sus características y condiciones de operación en el ámbito económico y productivo del país, las pocas normas legales relacionadas para este sector datan de 1973, año el cual se dictó la Ley de Fomento de la Pequeña Industria. Esto ha dificultado el desarrollo e implementación eficiente de políticas públicas adecuadas que fomenten el potencial productivo del sector industrial.

El desarrollo e impulso del sector de las pymes industriales parte de contar con un marco legal sólido, del que se desprenden las políticas necesarias para su activa participación en el proceso productivo del país, por lo que es escencial consolidar una base legal específica, sostenible y actualizada que responda a las necesidades competitivas y productivas del sector pyme.

Es necesario crear el entorno adecuado que promueva el fortalecimiento de las pymes a través la estabilidad económica y políticas que fomenten el potencial productivo de las mismas, en el campo financiero es indispensable contar con un sistema seguro que facilite la intermediación financiera eficiente relaizando canalización el ahorro hacia la inversión productiva., permitiendo acceder a crédito productivo a tasas de interés y condiciones razonables. 


\section{Bibliografía}

Armas, V. (1999). Esplendor y miseria de las pymes venezolanas. Revista Debates IESA 36 - 41

Cámara de Comercio de Quito. (2017). Clasificación de las Pymes, pequeña y mediana empresa (Vol. 1). Quito.

Codificación, C. L. y. Ley de Compañias, Codificación, 23 § (1999). Ecuador.

García-Moncó, A. (2011). La empresa familiar en la Ley de Sociedades de Capital: implicaciones fiscales. Pecunia: revista de la Facultad de Ciencias Económicas y Empresariales, 12(12), 119-132. https://doi.org/10.18002/pec.v0i12.618

GRECO, Silvia, "Cooperación internacional para pequeñas y medianas empresas", PYMES: escenario de oportunidades en el siglo XXI, Caracas, Venezuela, SELA, AECI, 1999.

SANCHEZ, Jeaneth, Elementos de Discusión hacia una política de la micro y pequeña empresa, Editorial La tierra, Quito, 2005 